\title{
Composite control of the SiC arc welding power source based on the expert system and neuron PID
}

\author{
Chen Si-min ${ }^{1}$, Zhong Qi-ming ${ }^{2}$, Zeng Zhi-jing ${ }^{1}$, Wang Zhen-min ${ }^{2 *}$ \\ 1. School of Electrical Technology, GuangDong Mechanical \& Electrical Polytechnic, GuangZhou, 510550; \\ 2. School of Mechanical and Automotive Engineering, South China University of Technology, GuangZhou, 510640
}

\begin{abstract}
A third generation wide-band-gap $\mathrm{SiC}$ semiconductor device is used in the $\mathrm{SiC}$ arc welding power source, which has a higher inverter frequency and greatly improves the dynamic characteristics of the arc welding power source, providing opportunities for control algorithm optimization. A composite control method of the arc welding power source combining the expert system and single neuron Proportional-Integral-Derivative (PID) is proposed in this paper, aimed at the fact that the proportional cofficient of neuron PID can hardly be adapted to rapid welding current changes. The SiC arc welding power source is taken as the plant of study in this paper. A mathematical model of the arc welding power source-arc system is established, and the controller of the arc welding power source based on the neuron PID and corresponding expert rules are defined to adjust the proportional coefficient of neuron PID Finally, the neuron PID controller (SNC) and the composite controller based on the expert system and neuron PID (ESNC) are simulated and verified. The results show that compared with the neuron PID algorithm, this method can adjust the proportional neuron PID coefficient in real time according to the welding current and has a better adaptive ability and superior tracking performance for arc welding power source control.
\end{abstract}

\section{Introduction}

Theband gap of a $\mathrm{SiC}$ power device is much wider than that of a Si-based power device, and the switching speed is higher, which provides better high-frequency characteristics for the arc welding power source. Based on analysis of the switching waveform and loss data in double-pulse tests by Hazra, it can be concluded that $\mathrm{SiC}$ power devices have more advantages than Si-based power devices in both the switching speed and loss [1]. The differences between Si-based and $\mathrm{SiC}$ power devices under the same conditions in high-frequency switching applications are compared in reference [2], and at present, the switching loss of a $\mathrm{SiC}$ power device is smaller than that of the best Si-based power device. A new generation of wide-bandgap welding power sources based on $\mathrm{SiC}$ power devices have been developed, as reported in reference [3], whose inverter frequency can be as high as $200 \mathrm{kHz}$; a full-bridge resonant converter with a new SiC power device, whose main circuit adopts an LLC topology structure and whose resonant converter frequency ranges from $260-$ to $310 \mathrm{kHz}$, has been established in reference [4]. The wide-bandgap welding power source has greatly improved the dynamic characteristics of the hardware platform of high-power arc welding power sources $[5,6]$. To fully utilize the hardware platform performance of the new ultrahigh-frequency $\mathrm{SiC}$ arc welding power source, an advanced control algorithm is needed. Currently, the incremental PIDcontrol algorithm is widely used in welding power control because of its simple principle and convenient use [7]. However, once the conventional incremental PID parameters are adjusted, they will remain unchanged in the whole control process, which is not conducive to tracking the set value and adapting to changes in welding process characteristics.

Many scholars have studied a variety of improved PID algorithms. It is pointed out in reference [8] that when the working area is frequently disturbed, artificial intelligence technology such as neural networks, fuzzy control or expert controllers can be used to optimize the adaptive welding parameters in real time and significantly reduce welding defects. Reference [9] adjusts the proportional coefficient in the incremental PID algorithm to achieve a rapid response through fuzzy immune PID control based on the biological immune feedback adjustment strategy and fuzzy logic reasoning. Zhang Liling uses the welding expert system of the Newton interpolation algorithm to realize self-adjustment of the process parameters of the power supply for cold welding of aluminum magnesium alloy sheets [10]. The application of a neuron PID controller with a strong robustness and adaptability is beneficial to further improve the control effect on an arc welding power source [11]. The simulation results in reference [12] reveal that the pulse current of the welding power source operated by a single neuron adaptive PID controller has a low intensity when the peak and base values are superposed. However, there are still shortcomings in the

Corresponding author email: 734380844@qq.com 
neuron PID control approach, such as a long adjustment time and difficulty in optimizing the proportional coefficient of neurons. There are few neuron PID improvement algorithms that can be applied to ultrahigh inverter frequency arc welding power sources.

\section{Problem description}

\subsection{Principle of the wide-bandgap SiC arc welding power source control system}

A block diagram of the wide-band-gap $\mathrm{SiC}$ arc welding power source control system is shown in Figure 1. The wide-band-gap $\mathrm{SiC}$ arc welding power source control system consists of a power module, welding voltage and current sampling and modulation circuits, human-computer interaction interface, ARM Cortex-M4 digital control processor, wide-bandgap power device driver module, wire feed control module, etc. The control principle is as follows: the ARM Cortex-M4 digital control processor samples and filters the electric signal detection circuit of the welding arc through the Analog-to-Digital (ADC) module, and the value is compared with the given value to generate a deviation; the controller uses the deviation to adjust the Pulse Width Modulation (PWM) duty cycle of the timer output, and the driving signal obtained through the wide-band-gap power device driving module isolation and amplification can control the conduction time of the power switch of the inverter power source and accurately adjust the output energy of the power source so that the arc current tends to remain stable near a given value.

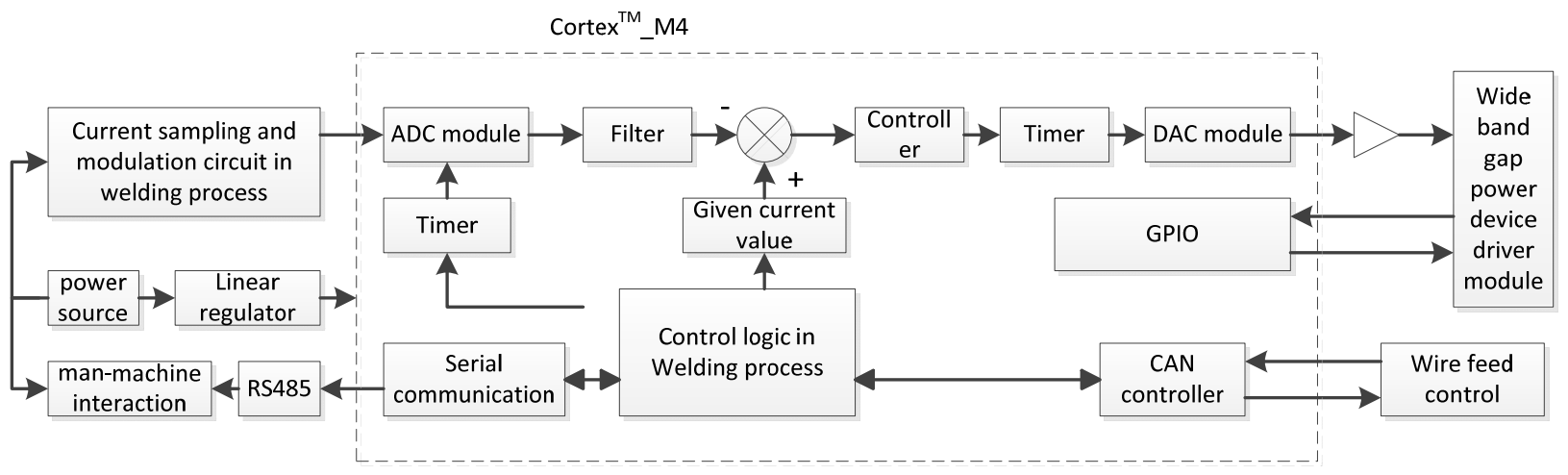

Fig. 1 Hardware diagram of the wide-band-gap SiC arc welding power source control system

\subsection{Mathematical model establishment of the arc welding power control system}

The arc welding power control system model consists of an arc welding power source-arc system, a digital PID controller and a feedback link. And arc welding power source-arc systems include inverter power sources and arc loads. The hardware platform of the arc welding power source adopts the wide-band-gap SiC arc welding inverter power source independently researched and developed in the laboratory, whose inverter frequency can reach $200 \mathrm{kHz}$; its dynamic response is rapid, and the energy adjustment period is shorter. The output $u(k)$ ranges from 10 to 375 , and is related to the digital PWM output of the control chip, which determines the PWM duty cycle, and the range of the voltage for arc welding power source is from 0 to $70 \mathrm{~V}$. The inverter link can be regarded as a purely delayed amplification link, which is caused by the out-of-control time constant of the inverter link. The transfer function is thus approximated.

$$
G_{V}(s)=\frac{K_{V}}{t_{V} s+1}
$$

where $K_{V}=\frac{U_{e 0}}{P W M}=\frac{70}{375}=0.19$, and

$t_{V}=10 \mu s$.
The full-wave rectifier module of the secondary side of the inverter power source forms a closed loop through the filter inductor, $L_{0}$, the internal resistance of the power supply, and the resistance of the output cable, $R_{0}$, and the arc load. Among them, $L_{0}=1 \times 10^{-5} H$, and $R_{0}=0.1 \Omega$. Its voltage balance equation is:

$$
U_{\text {eo }}(t)-U_{\text {out }}(t)=L_{0} \frac{d I(t)}{d(t)}+R_{0} I(t)
$$

After Laplace transformation, the equation becomes:

$$
G_{L R}(s)=\frac{K_{R}}{t_{L} s+1}
$$

where $K_{R}=\frac{1}{R_{0}}=10$, and $t_{L}=\frac{L_{0}}{R_{0}}=100 \mu \mathrm{s}$.

Therefore, the transfer function of the arc welding power source-arc system is:

$$
G(s)=G_{V}(s) \bullet G_{L R}(s)
$$

\section{Arc welding power controller based on the expert system and neuron PID}

Figure 2 shows the controller based on the expert system and neuron PID (ESNC), which further integrates the 
expert system based on the neuron PID control structure. According to the change in the feedback deviation, the proportional coefficient of the neuron PID is adjusted online, and the output is controlled, while the PWM duty ratio is adjusted to achieve fine control of the output current. At the same time, when the arc characteristics change, the proportional coefficient of the neuron PID can be adaptively adjusted so that the system can obtain a fast response with a lower overshoot..

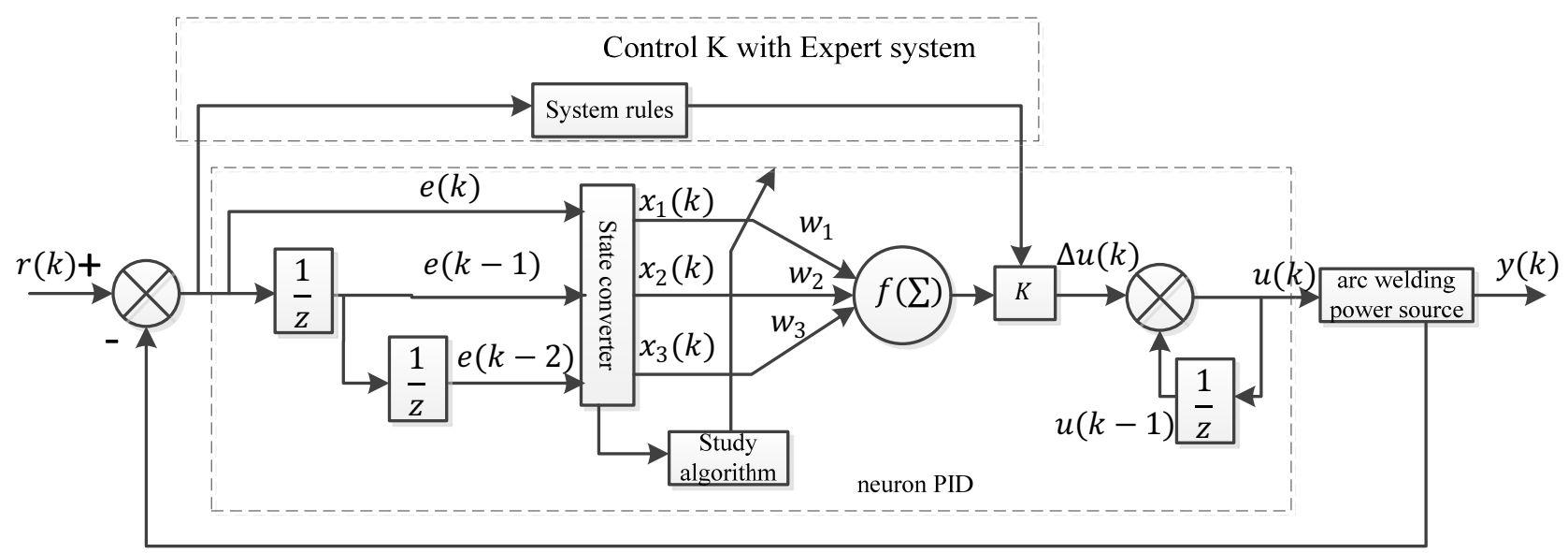

Fig. 2 Block diagram of the wide-band-gap SiC arc welding power source controller based on the expert system and single neuron PID

\subsection{Neuron PID control structure}

Neural network intelligent control technology has been applied in high-power power systems [11]. Among the various applications, neuron PID control is a combination of conventional incremental PID control and the neuron structure, and its control equation is:

$$
\begin{aligned}
\Delta u(k) & =K \sum_{i=1}^{3} w_{i}^{\prime}(k) x_{i} \\
& =K\left\{w_{1}^{\prime}(k)[e(k)-e(k-1)]+w_{2}^{\prime}(k) e(k)\right\} \\
& \left.+K \cdot w_{3}^{\prime}(k)[e(k)-2 e(k-1)+e(k-2)]\right\}
\end{aligned}
$$

The neuron is composed of three inputs and one output. The input layer includes $x_{1}(k)=e(k)-e(k-1), \quad x_{2}(k)=e(k), \quad$ a n d $x_{3}(k)=e(k)-2 e(k-1)+e(k-2)$, and the output layer consists of node $\Delta u(k)$, where $K$ is the pr oportionality coefficient, $w_{i}(k)$ is the weight coeffic ient of $x_{i}(k)$, and $w_{i}^{\prime}(k)=w_{i}(k) / \sum_{i=1}^{3}\left|w_{i}(k)\right|$.

In this paper, the supervised Hebb weight coefficient learning rule is used to update the weight coefficient to improve the robustness and performance of the controller and promote convergence of the learning algorithm. The equation is as follows:

$$
\begin{aligned}
& w_{1}(k)=w_{1}(k-1)+\eta_{p} e(k) u(k-1) x_{1}(k) \\
& w_{2}(k)=w_{2}(k-1)+\eta_{i} e(k) u(k-1) x_{2}(k) \\
& w_{3}(k)=w_{3}(k-1)+\eta_{d} e(k) u(k-1) x_{3}(k)
\end{aligned}
$$

where $\eta_{p}, \eta_{i}$, and $\eta_{d}$ are the learning rates whose value range is $[0,1]$.

The value of the proportional coefficient is closely related to the dynamic response and stability of the system: And the welding process has nonlinear and time-varying characteristics. The system needs to be adaptively adjusted according to the relative error of the welding process to achieve better stability and speed.

\subsection{Adjustment of the expert rules}

To improve the system response speed, the value of $K$ is considered in this paper. According to the absolute value of the standard deviation of the closed-loop response, $|\operatorname{er}(k)|$, the response is divided into several intervals based on expert knowledge, and the value of $K$ is different in each interval, which is modified according to the variation characteristic of the deviation within a certain range to reduce overshoot and adjustment time. This paper adopts production representation based on rules, which is adjusted by the IF-THEN structure. According to many experiments, the adjustment rules are as follows:

$$
\begin{aligned}
& \text { if } 0.8<|\operatorname{er}(k)| \leq 1 \text { then } K=a_{0}+b_{0}|\operatorname{er}(k)|^{3} / r(k) \\
& \text { if } 0.3<|\operatorname{er}(k)| \leq 0.8 \text { then } K=a_{1}+b_{1}|\operatorname{er}(k)| / 2 r(k) \\
& \text { if } 0.1<|\operatorname{er}(k)| \leq 0.3 \text { thenK }=a_{2}+b_{2}|\operatorname{er}(k)| / r(k) \\
& \text { if } 0<|\operatorname{er}(k)| \leq 0.1 \text { thenK }=a_{3}+b_{3}|\operatorname{er}(k)| / r(k)
\end{aligned}
$$

where $e r(k)=(r(k)-y(k))) f r(k)$ is the unitary deviation.

As shown in Equation 7, the regulation strategy of $K$ is as follows: at the initial stage of system response, a larger value of $K$ is used to enable the system to 
quickly reach a given value. Once the proportional coefficient continues to be large, the rise time will decrease, but overshoot will increase. To restrain system overshoot, the value of $K$ is reduced to ensure system stability and reduce overshoot and the adjustment time in the second stage. The third and fourth stages are to optimize the learning function of the neuron PID in the refined adjustment process that tends to be steady state. The value of $K$ is controlled by expert rules, considering both the fast and stable requirements of the system response. Among them, $a$ is used for approximate tuning, and $b$ is used for fine-tuning.

\section{Simulation and analysis}

The model is built in MATLAB, and both the neuron PID controller (SNC) and ESNC are simulated. The sampling period is $10 \mu \mathrm{S}$. The proportional coefficient of SNC is
$K=0.01$, the initial learning rates are $\eta_{p}=0.1$, $\eta_{i}=0.1$, and $\eta_{d}=0.1$, and the initial weight coefficients are $w_{1}(0)=0.26, w_{2}(0)=0.05$, and $w_{3}(0)=0.08$. The ESNC parameters are $a_{0}=0.04$, $b_{0}=0.4, a_{1}=0.005, b_{1}=0.02, a_{2}=0.02$, $b_{2}=0.001, a_{3}=0.015$, and $b_{3}=0.001$. The initial learning rates and weight coefficients are the same as above.

The system has a step response at the current setpoint of $300 \mathrm{~A}$, and at $t=0.00[S$, when wire sticking occurs during welding, the arc load changes, whose transfer function is updated to the following:

$$
G_{L R 2}(s)=\frac{11}{1.1 \times 10^{-4} s+1}
$$

The simulation curve is shown in Figure 3.

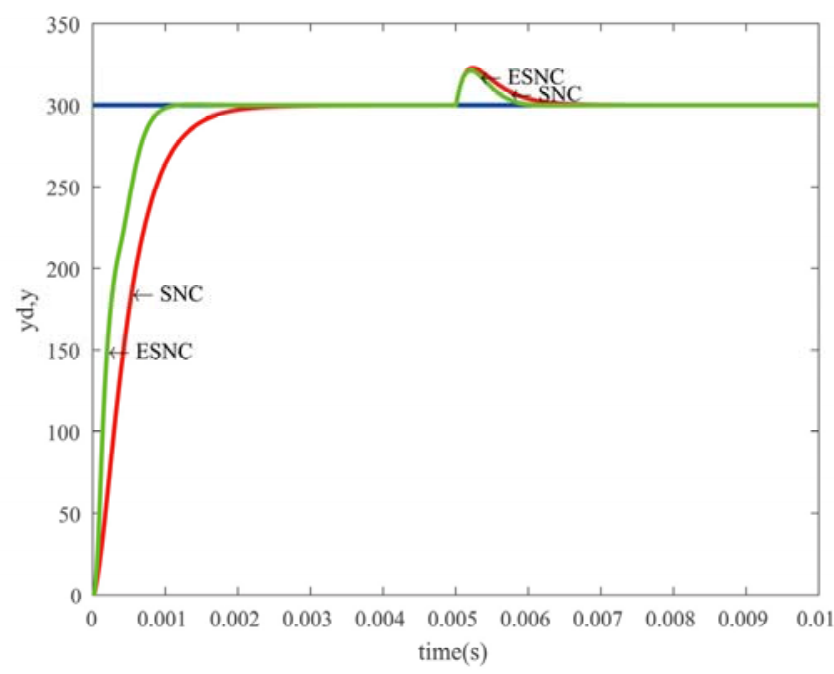

Fig. 3 Response curve of the welding current

Curve comparison shows that the overshoot of ESNC is $<1.5 \%$, and its performance is good. The ESNC system is more stable and faster than the SNC system, and the adjustment time of ESNC is $0.64 \mathrm{~ms}$, which is $47 \%$ less than that of SNC. The current can rise when wire sticking occurs during welding, and the system oscillates. The output is closer to the original given value of $300 \mathrm{~A}$, which is attained more smoothly and faster by ESNC compared with SNC, and the overshoot is reduced by $6 \%$, and the adjustment time is decreased by $38 \%$ in ESNC. Therefore, the composite control of a wide-bandgap SiC arc welding power source based on the expert system and neuron PID is feasible. The robustness and adaptability of the control system and the tracking performance under the condition of changing arc characteristics are greatly improved.

\section{Conclusion}

1) At a given current value, the arc welding power source controller based on the expert system and neuron PID can adjust the weight coefficient by the supervised Hebb learning algorithm and can adjust the proportional coefficient in four intervals based on expert rules, which results in the rise time of the welding current and overshoot being reduced.

2) The composite arc welding power controller based on the expert system and neuron PID has the advantages of a strong robustness, good adaptability and easy setting. When wire sticking occurs during welding, the composite controller based on the expert system and neuron PID can modify the proportional coefficient of the neuron PID dynamically based on expert rules, thereby enabling the system to be easily adapted to arc load changes with a shorter adjustment time and less overshoot, which is beneficial to improving the stability and response speed of the system. 


\section{Acknowledgement}

Fund projects: Key scientific research platforms and projects in Guangdong Universities(2018GkQNCX045); National Natural Science Foundation of China(51875212); Science and technology projects of Guangdong Province(2018A030313192); Guangzhou International Cooperation Project(201807010035 、 2019070110006)

\section{Reference}

1. Hazra S,De A,Cheng L, et al. High Switching Performance of $1700 \mathrm{~V}, 50 \mathrm{~A}$ SiC Power MOSFET over Si IGBT/BiMOSFET for Advanced Power Conversion Applications[J]. IEEE Transactions on Power Electronics, 2016, 31(7): 4742-4754.

2. Glaser J S,Nasadoski J J,Losee P A, et al. Direct comparison of silicon and silicon carbide power transistors in high-frequency hard-switched applications[C].// Applied Power Electronics Conference \& Exposition(APEC). Fort Worth, TX: IEEE, 2011: 1049-1056.

3. Wang Zhenmin, Wang Qian, et al. A new generation of WBG arc welding inverter power source[J]. Transactions of the China Welding Institution, 2016, 37(7): 49-52.

4. Wang Zhenmin, Wu Jianwen, Fan Wenyan, et al. Resonant soft switch plasma power source based on $\mathrm{SiC}$ MOSFET[J]. Journal of South China University of Technology(Natural Science edition), 2019, 47(1): 7-12.

5. Kar A,Ahmad S S,Narayanan G, et al. Design, performance evaluation, fabrication and testing of a $\mathrm{SiC}$ MOSFET gate driver[C].// 2017 IEEE International Conference on Signal Processing, Informatics, Communication and Energy Systems (SPICES). Kollam, India: IEEE, 2017: 6-6.

6. Machado R C S,Filho B J C. Next generation arc welding machines based on silicon carbide mosfets and high frequency planar magnetics[C].// 2015 IEEE 13th Brazilian Power Electronics Conference and 1st Southern Power Electronics Conference. New York: IEEE, 2015.

7. Wang Zhenmin, Xie Fangxiang, Feng Yunliang, et al Local dry welding system for underwater $\operatorname{robot}[\mathrm{J}]$, Transactions of the China Welding Institution, 2017,38(1):5-8.

8. Mvola B,Kah P,Martikainen J, et al. Control and Optimization of Output Current and Voltage Parameters: Effects on Joints Welded by Adaptive GMAW[C].// 20th International Scientific Conference on Mechanika 2015. Kaunas, Lithuania: Mechanika Kaunas University of Technology, 2015: 194-199.

9. Wang Zhenmin, Zhang Fubiao, Wang Pengfei, et al.VPPA welding power supply for full-digital robot[J]. Transactions of the China Welding Institution, 2017, 38(7):5-8,30.

10. Zhang L,Li B,Ye J. Power Supply and Its Expert System for Cold Welding of Aluminum and Magnesium Sheet Metal[C].// Intelligent Computing Methodologies. 15th International Conference, ICIC 2019. Nanchang, China: Springer International Publishing, Cham, Switzerland, 2019: 795-804.

11. Xu M,Xue J X. Control of Current Waveform for Pulsed MIG Welding of Aluminum Alloy Sheets[C].// Transactions on Intelligent Welding Manufacturing, Vol I, No. 2 2017. Shanghai, China: Springer-Verlag Singapore Pte Ltd, 2018: 163-172.

12. Changwen D,Jiaxiang $\mathrm{X}, \mathrm{Yu} \mathrm{H}$. Control Study of Pulsed MIG High-Speed Welding Power Source Based on Single Neuron Adaptive PID Model[C].// 2018 10th International Conference on Intelligent Human-Machine Systems and Cybernetics. Hangzhou, China: IEEE Computer Society, 2018: 381-5. 